Document downloaded from:

http://hdl.handle.net/10251/29276

This paper must be cited as:

Bhawe, Y.; Moliner Marin, M.; Lunn, JD.; Liu, Y.; Malek, A.; Davis, M. (2012). Effect of cage size on the selective conversion of methanol to light olefins. ACS CATALYSIS. 2:2490-2495. doi:10.1021/cs300558x

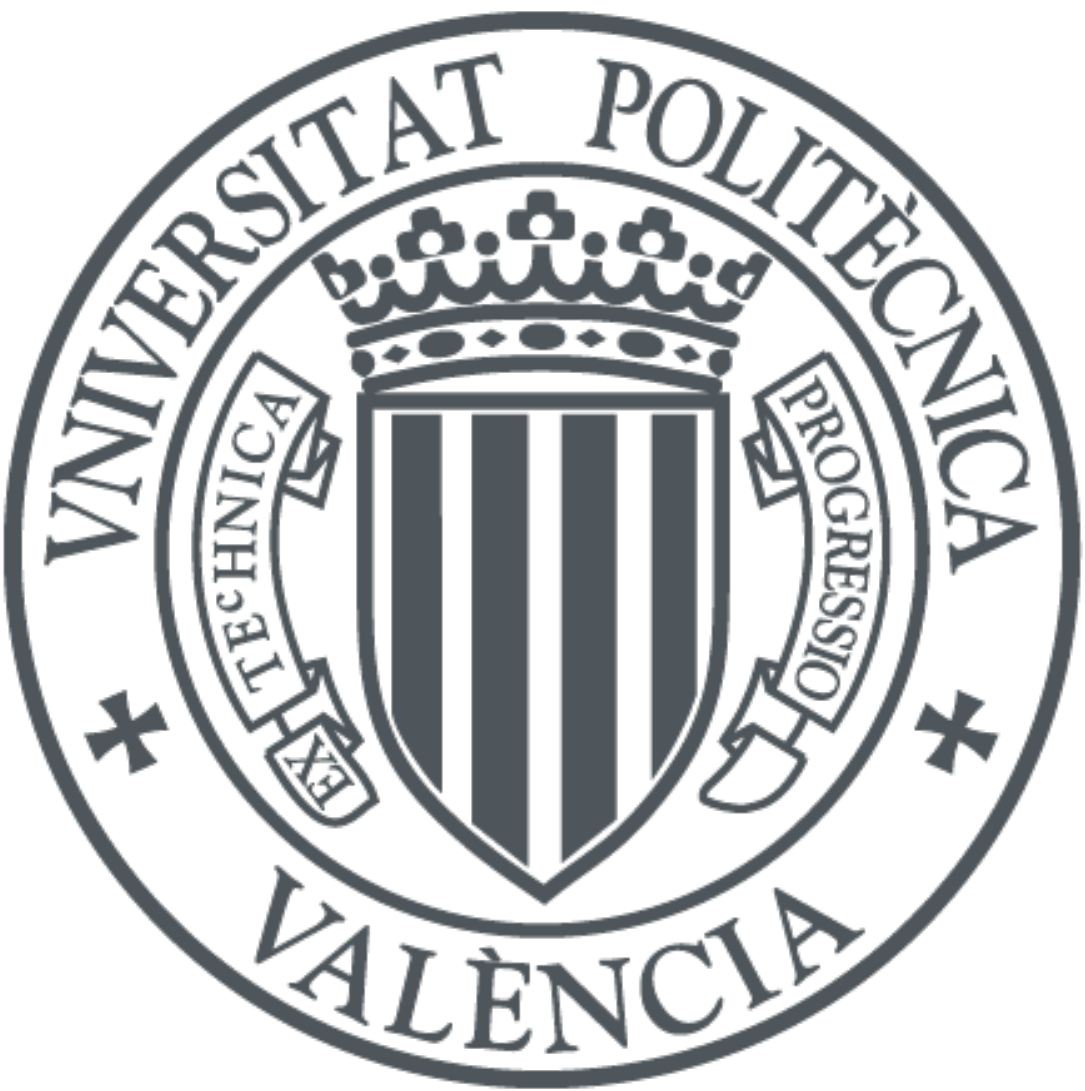

The final publication is available at

http://dx.doi.org/10.1021/cs300558x

Copyright American Chemical Society

Additional Information 
Title: Effect of cage size on the selective conversion of methanol to light olefins

Author List: Yashodhan Bhawe ${ }^{1}$, Manuel Moliner-Marin ${ }^{1 \#}$, Jonathan D Lunn ${ }^{2}$, Yu Liu ${ }^{3}$, Andrzej Malek ${ }^{2}$ and Mark Davis ${ }^{1}{ }^{*}$

${ }^{1 *}$ Corresponding Author: mdavis@cheme.caltech.edu

\section{Institution Addresses:}

1: Chemical Engineering, California Institute of Technology, Pasadena CA 91125

2: Inorganic Materials \& Heterogeneous Catalysis, The Dow Chemical Company, Midland MI 48640

3: Hydrocarbon and Energy R\&D, The Dow Chemical Company, Freeport TX 77541

Manuscript type: Article

\#Current Address: Instituto de Tecnología Química, UPV-CSIC, Universidad Politécnica de Valencia, Consejo Superior de Investigaciones Científicas, Valencia, Spain 46022 


\section{Supplementary Information}

\section{Scanning Electron Microscopy of synthesized samples}

Scanning electron microscopy/energy dispersive spectropscopy (SEM / EDS) analyses were conducted on a JEOL JSM-6700F instrument equipped with an Oxford INCA Energy 300 X-ray Energy Dispersive Spectrometer. The as-made powder samples were mounted on carbon tape prior to imaging.
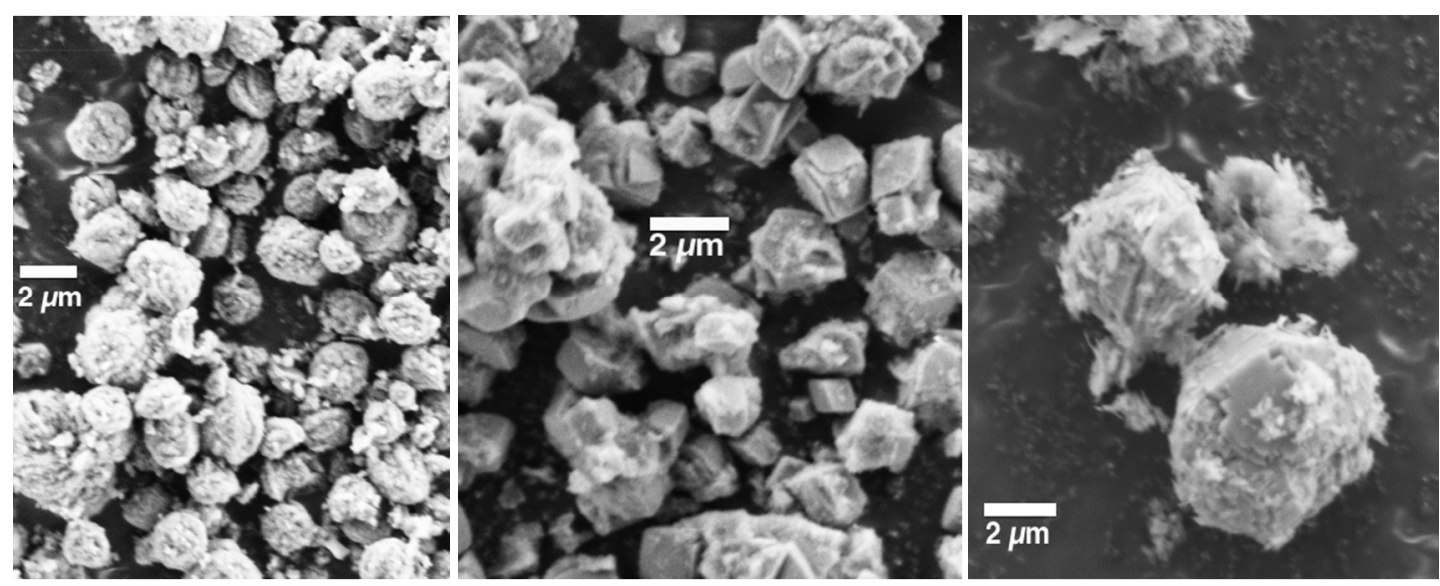

Figure S1: SEM Images of LEV (left), CHA (middle) and AFX (right)

\section{Magic Angle Spinning(MAS) NMR analysis of crystalline products}

MAS NMR was conducted on as-synthesized crystalline products. All spectra were taken on a Bruker Avance $500 \mathrm{MHz}$ spectrometer in $4 \mathrm{~mm} \mathrm{ZrO}_{2}$ rotors. On average, $60-80 \mathrm{mg}$ of sample were loaded per rotor. For ${ }^{27} \mathrm{Al}$ spectra, the rotor was spun at $14 \mathrm{kHz}$ with the ${ }^{27} \mathrm{Al}$ operating frequency tuned to $130.35 \mathrm{MHz}$. The ${ }^{29} \mathrm{Si}$ spectra were taken while the rotor was spinning at $8 \mathrm{kHz}$ with the operating frequency set to $99.37 \mathrm{MHz}$. 

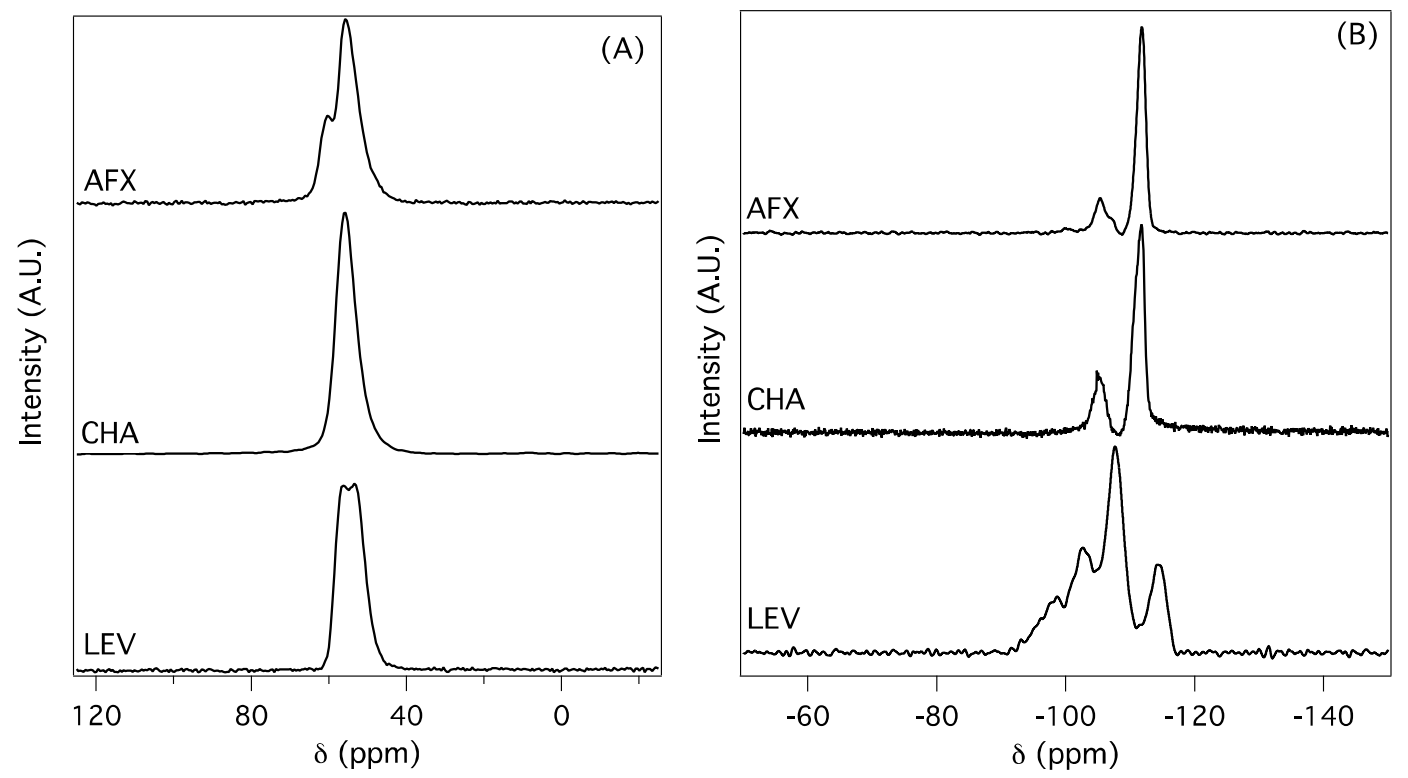

Figure S2: ${ }^{27} \mathrm{Al}$ (A) and ${ }^{29} \mathrm{Si}$ (B) MAS NMR spectra of as-made crystalline solids

\section{Additional Characterization of Occluded Organics: $U V$-vis and ${ }^{1} H$ NMR}

${ }^{1} \mathrm{H}$ NMR was conducted on a Varian $400 \mathrm{MHz}$ spectrometer using a standard ${ }^{1} \mathrm{H}$ NMR protocol. UV-Vis spectrophotometry was conducted on a Shimadzu UV-2401 spectrophotometer with samples diluted in chloroform (3 drops in $2 \mathrm{ml})$. 

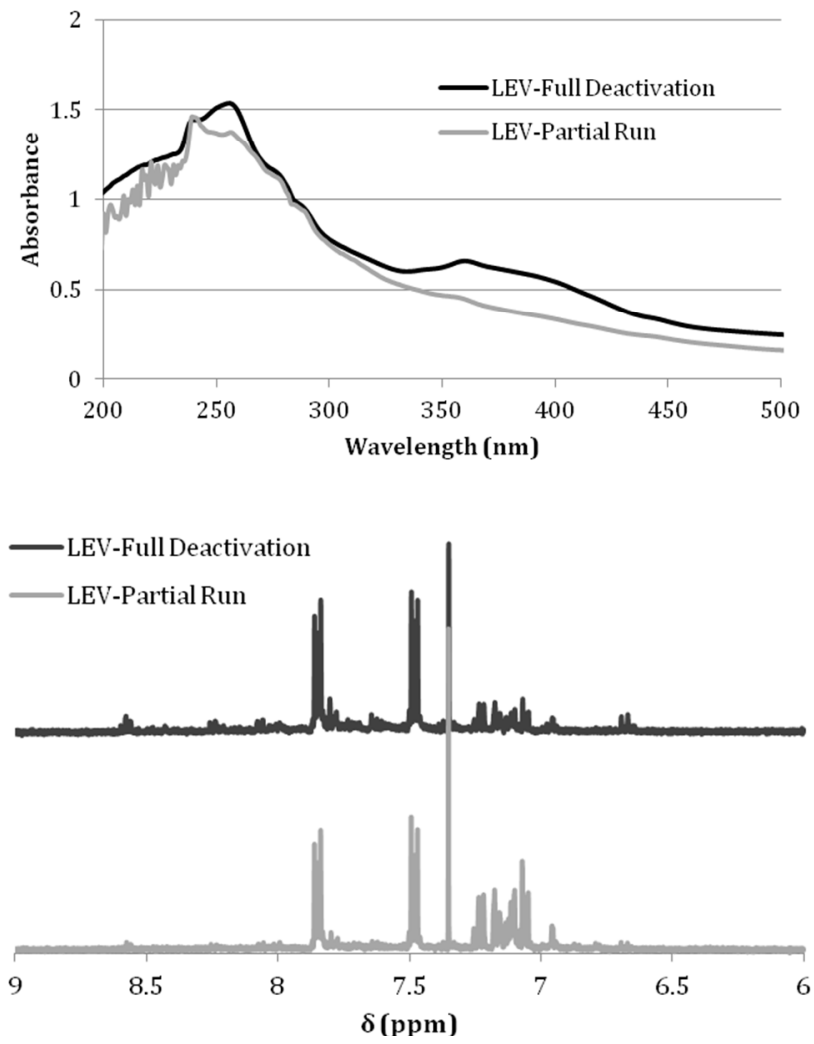

Figure S3 UV-visible absorption spectra (top) and ${ }^{1} \mathrm{H}$ NMR spectra (bottom) of the occluded organic species for LEV for fully and partially deactivated catalysts.

The increased level of anthrones observed in the GC/MS distribution is corroborated by a corresponding increase absorbance in the anthracene absorbance region (300-400nm) in the UV-vis (Figure S1). ${ }^{1} \mathrm{H}$ NMR for LEV shows the presence of unalkylated naphthalene (7.85 and $7.45 \mathrm{ppm}$, Figure S2) and toluene $(7.35 \mathrm{ppm})$ for the partially deactivated catalyst and fully deactivated zeolite as also seen in the GC/MS data. 

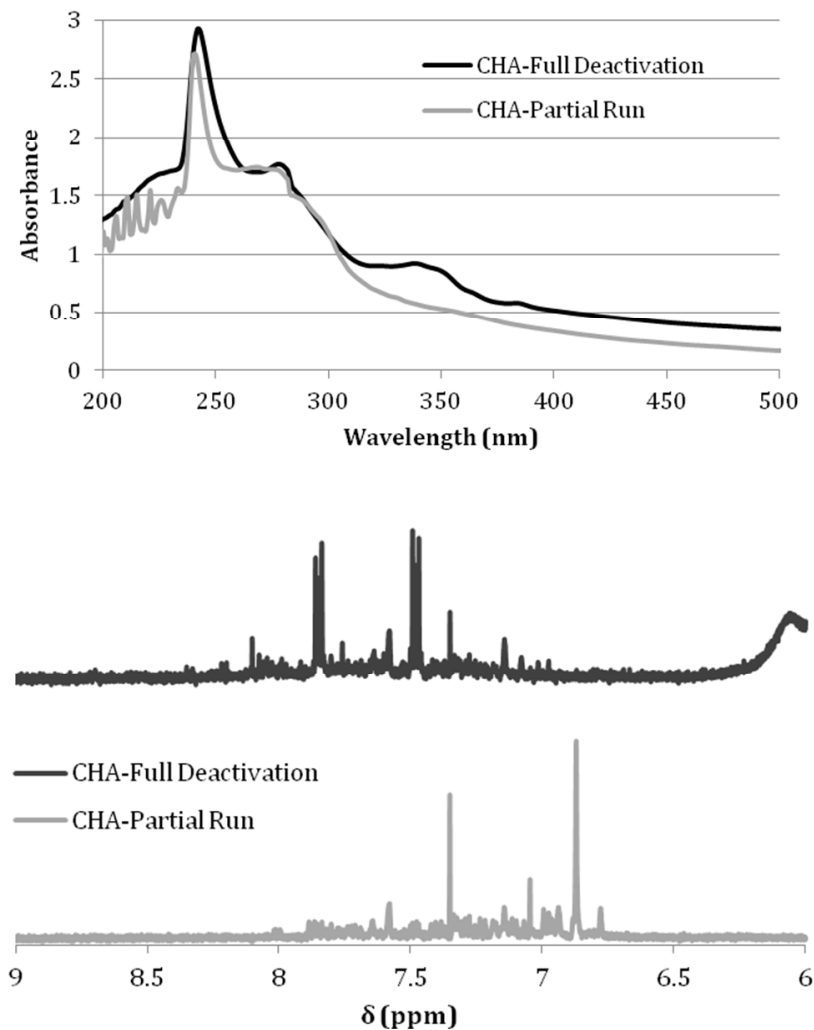

Figure S4 UV-visible absorption spectra (top) and ${ }^{1} \mathrm{H}$ NMR spectra (bottom) of the occluded organic species for CHA for fully and partially deactivated catalysts

The shift from alkylated benzenes to napthalenes in the fully deactivated CHA seen in the shifting distribution in the GC/MS is also seen in the displacement of alkylated benzene ${ }^{1} \mathrm{H}$ NMR chemical shifts $(6.7-7.4 \mathrm{ppm})$ to those of naphthalene (7.85 and $7.45 \mathrm{ppm})$. The small increase of anthracene species in the fully deactivated CHA is also evidenced in the in the UV-vis spectra $(300-400 \mathrm{~nm})$. 

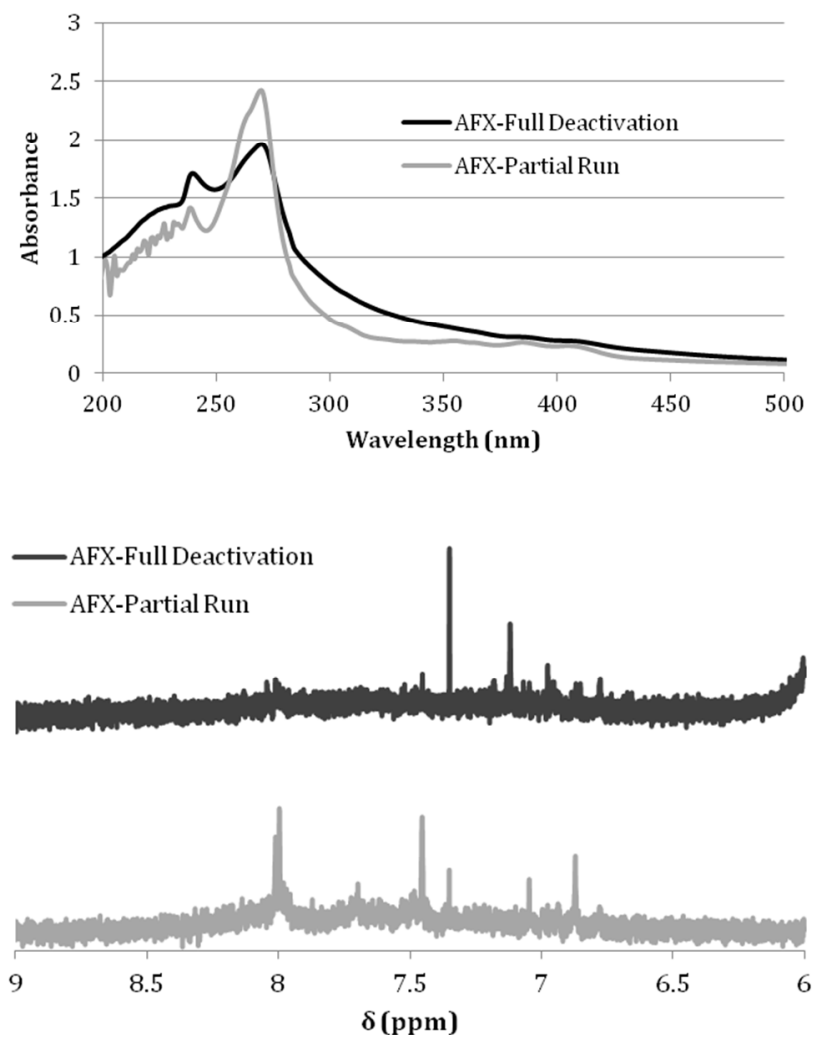

Figure S5 UV-visible absorption spectra (top) and ${ }^{1} \mathrm{H}$ NMR spectra (bottom) of the occluded organic species for AFX for fully and partially deactivatied catalysts.

In corroboration with the GC/MS results for AFX, the ${ }^{1} \mathrm{H}$ NMR spectra of the partial run zeolite shows some anthracene protons $(7.99$ and $7.45 \mathrm{ppm})$ that are then lost due to in the fully deactivated AFX, presumably by increased alkylation. The increase of alkylated benzene chemical shifts, including toluene $(7.35 \mathrm{ppm})$ is also observed in the fully deactivated AFX, which corresponds to the monoaromatic void filling suggested by the GC/MS. 